\title{
VALIDAÇÃO E APLICAÇÃO DE MÉTODO PARA ANÁLISE DE PESTICIDAS EM ÁGUA PARA CONSUMO HUMANO DE DOURADOS (MS) POR CLAEIUV E CGIDTE
}

\author{
MARA N. T. LOPES* \\ PEDRO TOLEDO NETTO** \\ CAROLINA LOURENCETTI*** \\ MARY R. R. DE MARCHI $I^{* * * *}$ \\ MARIA L. RIBEIRO*****
}

\begin{abstract}
O uso inadequado de pesticidas na agricultura tem sido apontado como fonte de risco para a saúde humana e para o ambiente. Considerando que os recursos hídricos são os principais destinos desses compostos após a aplicação, este trabalho apresenta a otimização e validação de dois métodos analíticos simples e eficientes para a determinação de pesticidas em águas superficiais e subterrâneas. Foram selecionados os pesticidas mais aplicados no município de Dourados, (Mato Grosso - Brasil) com intensa atividade agrícola. Efetuou-se a pré-concentração por extração em fase sólida com cartucho C18 (500 mg) e eluição com metanol para as amostras analisadas por cromatografia a líquido de alta eficiência com detector espectrofotométrico na região do ultravioleta (CLAE/ UV) (2,4-D e 2,4-DCF) e com acetato de etila:diclorometano (1:1, $\mathrm{v} / \mathrm{v}$ ) para as analisadas por cromatografia a gás com detector termiônico específico (CG/DTE) (atrazina, DIA, DEA, trifluralina e parationa metílica). Os métodos apresentaram exatidão (76-107\%) e precisão $(<12 \%)$ satisfatórias para as substâncias nos níveis de fortificação selecionados, exceto para DIA $(<51 \%)$, assim como para o estudo de estabilidade dos pesticidas $\left(-20^{\circ} \mathrm{C}\right.$ por até 21 dias). Os limites de quantificação dos métodos $\left(0,22-0,48 \mu \mathrm{g} \mathrm{L}^{-1}\right)$ estão de acordo com a legislação brasileira vigente para pesticidas em água. Embora somente o 2,4-D tenha sido detectado em dois pontos de coleta no período estudado, alerta-se para a necessidade de avaliação sistemática da presença de pesticidas em água para consumo humano, particularmente, em regiões com intensa atividade agrícola. Tal monitoramento pode fornecer subsídios para políticas públicas ambientais.
\end{abstract}

PALAVRAS-CHAVE: VALIDAÇÃO; PESTICIDAS; CLAE/UV; CG/DTE; ÁGUA - CONSUMO HUMANO.

* Doutora em Química, Professora, Faculdade de Ciências Biológicas e Ambientais, Universidade Federal da Grande Dourados (UFGD), Dourados, MS (e-mail: mntlopes@hotmail.com).

** Doutorando em Química, Instituto de Química, Universidade Estadual Paulista (UNESP), Araraquara, SP (e-mail: ptolledo@yahoo.com.br).

*** Doutora em Química, Professora, Instituto Federal de Educação, Ciência e Tecnologia de São Paulo (IFSP), Matão, SP (e-mail: carollourencetti@yahoo.com.br).

**** Doutora em Química, Professora, Instituto de Química, UNESP, Araraquara, SP (e-mail: mssqam@ iq.unesp.br).

***** Doutora em Química, Professora, Programa de Mestrado em Desenvolvimento Regional e Meio Ambiente, Centro Universitário de Araraquara (UNIARA), Araraquara, SP (e-mail: mariabiluribeiro@gmail.com). 


\section{INTRODUÇÃo}

Aproximadamente dois terços da superfície do planeta terra é coberta por água. Entretanto, apenas cerca de $0,6 \%$ estão acessíveis para o consumo humano, porcentagem essa representada pelas águas dos rios, lagos e lençois freáticos (SPRINGWAY, 2005). O consumo de água nas atividades humanas varia muito entre regiões e países. Os usos múltiplos da água e a necessidade permanente de se enfrentar o crescimento o populacional e às demandas industriais e agrícolas têm gerado pressão sobre os recursos hídricos superficiais e subterrâneos (TUNDISI, 2003a). No Brasil, $59 \%$ da água doce são destinados à agricultura, $19 \%$ ao uso industrial e $22 \%$ ao uso doméstico (SPRINGWAY, 2005).

O crescimento populacional não só tornou a água escassa, mas criou a necessidade do aumento da produção de alimentos e, para isso, novas fronteiras agrícolas foram abertas (TUNDISI, 2003a). Exemplo particularmente importante envolve a implantação de monoculturas que têm sido apontadas como uma das principais responsáveis pela degradação do ambiente, devido alta dependência de insumos agrícolas, entre os quais os pesticidas. Segundo a Organização das Nações Unidas para a Agricultura e Alimentação, o Brasil é um dos países que mais consome pesticidas nas lavouras (ALVES FILHO, 2002). A região Centro-Oeste brasileira ilustra bem esse problema, o qual vem exercendo forte pressão sobre o ecossistema, particularmente, sobre os fatores que interferem na qualidade das águas. Os riscos de degradação hídrica são diversificados, destacandose a utilização generalizada de pesticidas nas grandes lavouras da região, principalmente de soja e milho, e a pequena quantidade de propriedades rurais com práticas de conservação do solo que poderiam amenizar o problema.

O uso inadequado de pesticidas na agricultura tem sido apontado como fonte de risco para a saúde humana e para o ambiente, pois os recursos hídricos superficiais ou subterrâneos são os principais destinos desses compostos (RIBEIRO et al., 2007; DORES e DE-LAMONICA-FREIRE, 2001). Embora a legislação brasileira inclua pesticidas entre os parâmetros de qualidade da água utilizada para consumo humano, nem todos os produtos registrados para uso são contemplados (BRASIL, 2006). O controle da qualidade da água utilizada para consumo humano deve ser considerado para gerenciamento efetivo dos recursos hídricos, visando conhecer seu estado, seu potencial e os possíveis problemas agregados de contaminação e poluição. O monitoramento constitui um dos primeiros passos para a elaboração de banco de dados confiável e adequado que possa ser útil ao planejamento e ao gerenciamento desses recursos (TUNDISI, 2003b).

Estudos de monitoramento apresentam elevado custo e consomem muito tempo (SPADOTTO et al., 2004). Considerando o grande número de amostras e princípios ativos que devem ser determinados, métodos multirresíduos confiáveis e sensíveis devem ser desenvolvidos para detectar limites máximos de resíduos (LMR) e concentrações consideradas de alerta para a saúde humana (SABIK e JEANNOT, 2000).

Como o conjunto de análises e informações sobre a qualidade da águas utilizadas para consumo humano possibilitam verificar tendências, avaliar impactos e subsidiar políticas públicas (TUNDISI, 2003b), este trabalho apresenta a otimização e a validação de dois métodos analíticos simples e eficientes para a determinação dos pesticidas mais aplicados no município de Dourados (MS), pertencente à microbacia do Rio Dourados, localizado na região Centro-Oeste do Brasil.

\section{MATERIAL E MÉTODOS}

\subsection{REAGENTES}

Padrões de ácido 2,4-diclorofenolacético (2,4-D) (99\%), atrazina (99\%), parationa metílica (98,5\%), 2,4-diclorofenol (2,4-DCF) (99,5\%), trifluralina (99,5\%) desisopropilatrazina (DIA) (96,5\%) 
e desetilatrazina (DEA) $(95,5 \%)$ foram obtidos de Dr. Ehrenstorfer (Augsburgo, Alemanha) (Figura 1). Soluções estoque (200 $\mathrm{mg} \mathrm{L}^{-1}$ ) e soluções de trabalho de 2,4-D e 2,4-DCF foram preparadas em metanol e os demais pesticidas, atrazina, DIA, DEA, parationa metílica e trifluralina em acetato de etila, e armazenadas a $-20^{\circ} \mathrm{C}$. Foram usados metanol, acetona, acetato de etila e diclorometano grau CLAE (Mallinckrodt), ácido fórmico (Synth) e formiato de amônio (Vetec) grau p.a. Cartuchos de extração em fase sólida (EFS) (C18, $500 \mathrm{mg}, 55 \mu \mathrm{m}, 70 \mathrm{~A})$ foram obtidos de Strata. Usou-se água deionizada grau CLAE de sistema de purificação de água Milliopore (Milford, MA, USA).

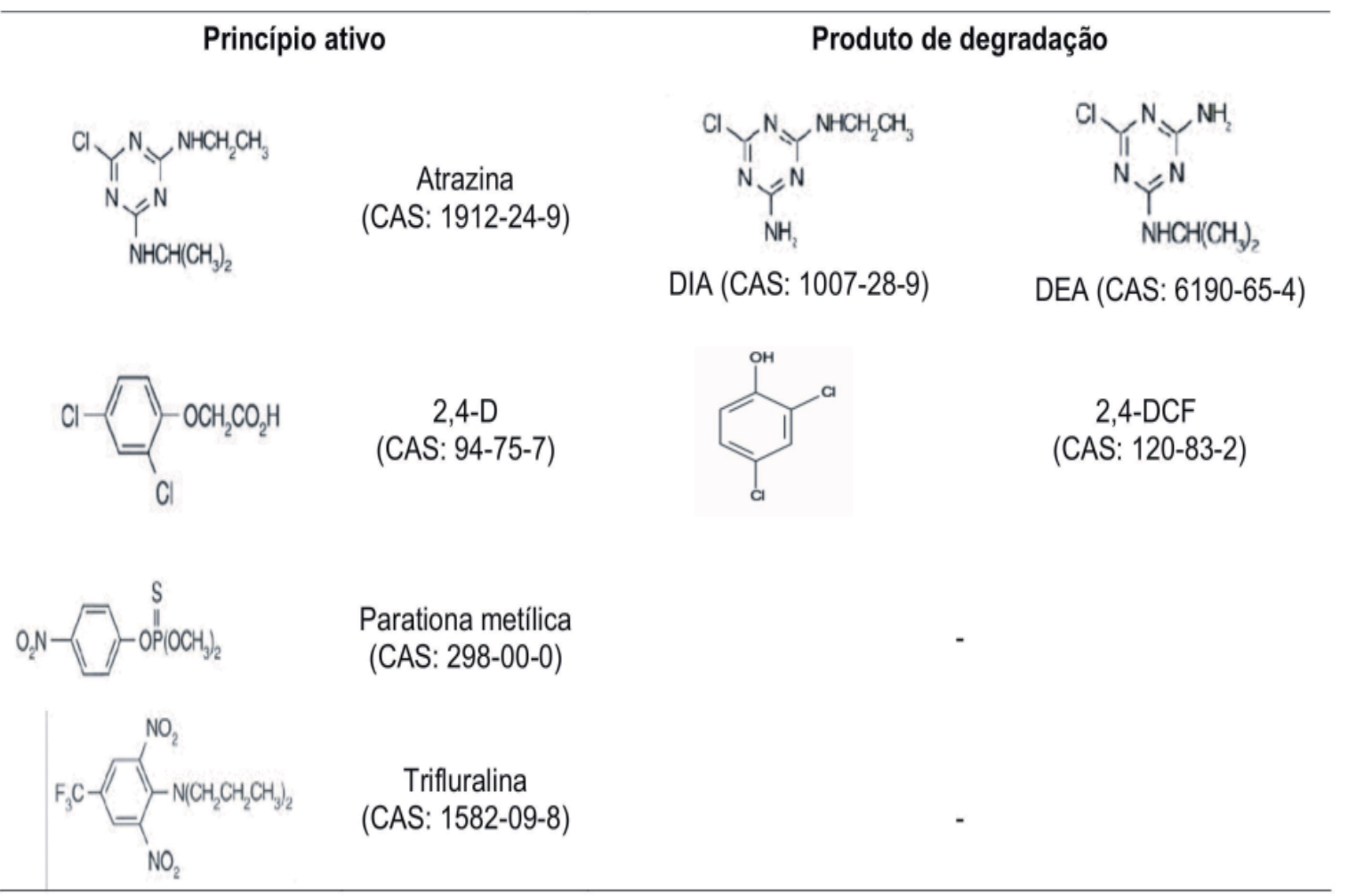

FIGURA 1 - ESTRUTURAS QUÍMICAS DAS SUBSTÂNCIAS ESTUDADAS

\subsection{DESENVOLVIMENTO E VALIDAÇÃO DO MÉTODO}

\subsubsection{Condições Cromatogáficas}

Dois sistemas cromatográficos foram utilizados neste estudo. Para análise dos pesticidas classificados como grupo I (atrazina, DIA, DEA, trifluralina e parationa metílica) usou-se cromatógrafo a gás Varian (Modelo 3300), equipado com detector termiônico específico (CG/DTE), acoplado a integrador Varian (Modelo 4290). A temperatura da coluna capilar de sílica fundida DB-5 (J\&W Scientific, $30 \mathrm{~m} \times 0,25 \mathrm{~mm}$ d.i. $\times 0,25 \mu \mathrm{m}$ espessura de filme) foi mantida a $140^{\circ} \mathrm{C}$ por 1 min e elevada até $180^{\circ} \mathrm{C}$ a $5^{\circ} \mathrm{C} \mathrm{min}{ }^{-1}$. Após $10 \mathrm{~min}$, aumentou-se a temperatura para $260^{\circ} \mathrm{C}$ a $5^{\circ} \mathrm{C} \mathrm{min}{ }^{-1}$ que foi mantida por $5 \mathrm{~min}$. Usou-se corrente da pérola de 3,4 A e as temperaturas do detector e injetor foram $320^{\circ} \mathrm{C}$ e $240^{\circ} \mathrm{C}$, respectivamente. Adotou-se o modo de injeção splitless/split (40s) e volume de $1 \mu \mathrm{L}$. Os fluxos dos gases foram: $4 \mathrm{~mL} \mathrm{~min}{ }^{-1}$ para hidrogênio, $175 \mathrm{~mL} \mathrm{~min}{ }^{-1}$ para ar sintético e $30 \mathrm{~mL} \mathrm{~min}^{-1}$ para nitrogênio. Usou-se cromatógrafo à líquido (Waters, Milford, MA, USA), equipado com sistema binário de bombas (Modelo 501), injetor manual com $20 \mu \mathrm{L}$ de capacidade (Rheodyne), detector espectrofométrico UV-Vis variável Waters (CLAE/UV) (Modelo 486) e integrador Varian (Modelo 4290) para determinar os pesticidas classificados como grupo II (2,4-D e 2,4-DCF). Usou-se coluna 
Lichrospher $100 \mathrm{RP} 18$ (Merck, $250 \mathrm{~mm} \times$ 4,6mm, $5 \mu \mathrm{m}$ ), conectada em pré-coluna Lichrospher 100 RP18 (Merck, $20 \mathrm{~mm} \times$ 4,6 mm, $5 \mu \mathrm{m}$ ), ambas de aço inoxidável, com volume de injeção de $20 \mu \mathrm{L}$. A fase móvel foi composta pela mistura metanol:tampão formiato de amônio $5,0 \mathrm{mmol} \mathrm{L}^{-1}$ $(60: 40, \mathrm{v} / \mathrm{v})$ a pH $4,5 \mathrm{em}$ modo de eluição isocrático $\left(0,7 \mathrm{~mL} \mathrm{~min}^{-1}\right)$. A fase móvel, preparada diariamente, foi filtrada em membrana de $0,45 \mu \mathrm{m}$ (Millipore, USA) antes do uso e desgaseificada em ultrassom. Os pesticidas foram determinados simultaneamente a $230 \mathrm{~nm}$ em temperatura ambiente.

\subsubsection{Fortificação das amostras e otimização do método}

Amostras de água (2 L) de poço artesiano, localizado nas dependências do Instituto de Química de Araraquara (UNESP), foram utilizadas para o desenvolvimento e validação dos métodos apresentados neste estudo. Após homogeneização e filtração (membrana 0,45 $\mu \mathrm{m}$ Sob vácuo), as amostras de água foram fortificadas com adição de solução dos padrões das pesticidas estudados. Para obtenção das amostras fortificadas com o grupo de substâncias I, $100 \mu \mathrm{L}$ da solução mista dos padrões foi adicionada em $500 \mathrm{~mL}$ da amostra de água. Para a obtenção das amostras fortificadas com o grupo de substâncias II, ajustou-se o pH de $200 \mathrm{~mL}$ da amostra de água para $2 \mathrm{com}$ ácido clorídrico concentrado e adicição de $100 \mu \mathrm{L}$ da solução mista dos padrões. Dois níveis de recuperação foram obtidos, 1 e $2 \mu \mathrm{gL}^{-1}$ para DIA, DEA, atrazina e parationa metílica, 10 e $20 \mathrm{\mu g} \mathrm{L}^{-1}$ para trifluralina e 10 e $30 \mu \mathrm{g} \mathrm{L}^{-1}$ para 2,4-D e 2,4-DCF.

Aotimização dos métodos para determinação das substâncias dos grupos I e ll em água teve como base trabalhos encontrados na literatura sobre a análise desses compostos nesse mesmo tipo de matriz (DALVIE et al., 2005; PAPADOPOULOU-MOURKIDOU et al., 2004a; PAPADOPOULOUMOURKIDOU et al., 2004b; AZEVEDO; GERCHON e REIS, 2004; RODRIGUES-MOZAZ, ALDA e BARCELÓ, 2004; MA et al., 2003; GONÇALVES e ALPENDURADA, 2002; PINTO e JARDIM, 2002; MENOR-HIGUERUELO et al., 2002; LAGANÀ et al., 2002; FILIZOLA et al., 2002; AMARANTE JUNIOR et al., 2002).

Testes preliminares em $500 \mathrm{~mL}$ de água foram efetuados para avaliar o condicionamento do cartucho (metanol e água, metanol, acetato de etila e água e acetona, metanol e água), a velocidade de eluição da amostra $\left(5,8\right.$ e $\left.10 \mathrm{~mL} \mathrm{~min}^{-1}\right)$, o tempo de secagem do cartucho após eluição da amostra $(15,30$ e $45 \mathrm{~min})$ e a eluição dos pesticidas (acetato de etila, acetato de etila:n-hexano (7:3, v/v) e acetato de etila:diclorometano $(1: 1, \mathrm{v} / \mathrm{v}))$. Tais testes visaram o estabelecimento de parâmetros experimentais adequados para a análise dos pesticidas do grupo I por EFS (C18) e CLAE/UV. A otimização do método para análise do 2,4-D e 2,4-DCF consistiu na avaliação da velocidade de eluição de $200 \mathrm{~mL}$ da amostra $\left(3,5\right.$ e $\left.8 \mathrm{~mL} \mathrm{~min}^{-1}\right)$ em cartucho C18, previamente acondicionado com metanol e água e na avaliação do tempo de secagem do cartucho (5, 30 e $60 \mathrm{~min})$ após a eluição da amostra.

O procedimento empregado para a validação do método de análise do grupo I consistiu no condicionamento do cartucho de extração em fase sólida com $10 \mathrm{~mL}$ de acetona, seguido por $10 \mathrm{~mL}$ de metanol e $10 \mathrm{~mL}$ de água deionizada $\left(8 \mathrm{~mL} \mathrm{~min}^{-1}\right)$. Conforme recomendações de Fritz (2005), a adição da amostra (500 mL) ao cartucho, previamente filtrada, foi realizada logo após seu condicionamento, evitando a secura. Adotou-se velocidade de pré-concentraçao de $8 \mathrm{~mL} \mathrm{~min}^{-1} \mathrm{e}$ tempo de secagem do cartucho de $30 \mathrm{~min}$. Utilizou-se a mistura acetato de etila:diclorometano (1:1, $\mathrm{v} / \mathrm{v}$ ) e duas alíquotas de $10 \mathrm{~mL}$ a $2 \mathrm{~mL} \mathrm{~min}{ }^{-1}$ para a eluição dos analitos. O eluato, concentrado até quase a secura em evaporador rotatório, foi retomado em 1,0 mL de acetato de etila e os analitos determinados por CG/DTE. Para a validação do método de análise do grupo II, acondicionaram-se cartuchos de EFS sequencialmente com $10 \mathrm{~mL}$ de metanol e $10 \mathrm{~mL}$ de água deionizada a $5 \mathrm{~mL}$ $\mathrm{min}^{-1}$. Após a eluição de $200 \mathrm{~mL}$ da amostra filtrada $\left(5 \mathrm{~mL} \mathrm{~min}^{-1}\right)$, o cartucho foi lavado com $5 \mathrm{~mL}$ de água e seco sob vácuo durante 30 minutos. Realizou-se a eluição dos analitos com alíquota de $3 \mathrm{~mL}$ de metanol e vazão de $2 \mathrm{~mL} \mathrm{~min}^{-1}$. Ajustou-se o volume final do eluato para $3,0 \mathrm{~mL}$, sendo os analitos determinados por CLAE/UV. 


\subsubsection{Validação do método}

Avaliou-se a eficiência dos métodos de análise dos dois grupos de analitos estudados, I e II, mediante estudo de recuperação de amostras de água fortificadas e não fortificadas (amostra testemunha) $(n=5)$, seguindo recomendações de Thier e Zeumer (1987) para análise de resíduos de pesticidas. A exatidão e a precisão dos resultados foram determinadas pelos valores de porcentagem de recuperação e do coeficiente de variação, respectivamente. Avaliou-se a especificidade das análises mediante análise de amostras de água sem adição dos padrões (amostra controle/ testemunhas).

Calcularam-se os limites de detecção (LD) e quantificação (LQ) dos métodos, segundo Thier e Zeumer (1987), considerando o menor nível de fortificação e as amostras testemunha.

\subsubsection{Estudo de estabilidade dos pesticidas}

Avaliou-se a estabilidade dos pesticidas pré-concentrados em cartuchos mantidos sob refrigeração $\left(-20^{\circ} \mathrm{C}\right)$ durante 7,14 e 21 dias antes da extração, mediante estudos de recuperação $(n=3)$ de amostras de água coletadas na Estação de Tratamento de Água da cidade de Araraquara (DAAE), fortificadas com os analitos dos grupos I e II, separadamente, nos dois níveis de fortificação estudados.

\subsection{APLICAÇÃO DO MÉTODO}

\subsection{1 Área de estudo}

A cidade de Dourados está localizada ao sul do estado do Mato Grosso do Sul, região Centro-Oeste do Brasil, a 458 m do nível do mar, sob as coordenadas $22^{\circ} 10^{\prime}$ de Latitude Sul e $54^{\circ} 56^{\prime}$ de Longitude Oeste. A sede de seu município está parcialmente inserida na bacia do Rio Dourados e $75 \%$ de sua água de abastecimento urbano provém de água superficial e $25 \%$ de água subterrânea (LOPES, 2001). Esse município apresenta intensa atividade agrícola, sendo as principais culturas o algodão, a soja e o milho. O uso de herbicidas predomina no preparo do solo, sendo os produtos aplicados no plantio e durante a capina da cultura. O tipo de solo predominante na região, Latossolo roxo, é pobre em matéria orgânica o que pode contribuir para a lixiviação dos pesticidas

\subsubsection{Coleta e tratamento das amostras}

O período das coletas das amostras compreendeu os meses de setembro de 2004 (estiagem e ausência da aplicação efetiva dos pesticidas), janeiro de 2005 (chuvas intensas e uso de pesticidas) e abril de 2005 (uso de pesticidas e final do período de chuvas), sendo as coletas efetuadas pela manhã por dois dias consecutivos. Amostras de água $(2 \mathrm{~L})$ foram coletadas em frascos âmbar (adequadamente limpos) diretamente da torneira, antes da caixa d'água, de residências próximas aos centros de distribuição de água da empresa de saneamento do Mato Grosso do Sul (Sanesul) (P1 e P4 mistura de águas superficial e subterrânea e P2 e P3 apenas água subterrânea) e da saída das bombas de poços artesanais particulares (região central da cidade (P5), núcleo industrial (P6), cidade universitária (P7) e propriedade rural (P8)). Em ambos os casos (residências e poços) coletaram-se as amostras de água após deixar as torneiras abertas e as bombas ligadas por 10 minutos. A temperatura e o $\mathrm{pH}$ de todas as amostras foram medidos no momento da coleta. Os frascos foram transportados para o laboratório da Universidade Federal de Mato Grosso do Sul (UFMS), Câmpus de Dourados, em caixas térmicas e armazenados em geladeira a $4^{\circ} \mathrm{C}$ até a pré-concentração em cartuchos de EFS, a qual foi realizada até três dias após a coleta. Depois da pré-concentração, os cartuchos foram transportados sob refrigeração $\left(4^{\circ} \mathrm{C}\right)$ até o Laboratório de Resíduos de Pesticidas do Instituto de Química de Araraquara (UNESP), sendo mantidos a $-20^{\circ} \mathrm{C}$ 
até a eluição e determinação. O intervalo entre a coleta, pré-concentração, transporte, extração e determinação dos pesticidas não ultrapassou 20 dias.

\section{RESULTADOS E DISCUSSÃO}

\subsection{SELEÇÃO DOS PESTICIDAS}

Os pesticidas selecionados para este estudo, atrazina e seus principais produtos de degradação (DIA e DEA), trifluralina e parationa metílica (grupo I) e 2,4-D e seu principal produto de degradação 2,4-DCF (grupo II), estão entre os mais utilizados na região da microbacia do Rio Dourados nas culturas de soja, milho e algodão. Também estão entre os que vêm sendo determinados em amostras de águas superficiais e subterrâneas no Brasil e em outros países, como Índia, Espanha, Grécia, Portugal e Itália (RODRIGUES-MOZAZ; ALDA e BARCELÓ, 2004; LAGANÀ et al., 2002; DORES et al., 2006; SANKARARAMAKRISHNAN, SHARMA e SANGHI, 2005; PALMA et al., 2009; LAMBROPOULOU et al., 2002; AZEVEDO et al., 2000; ALBANIS e HELA, 1995).

\subsection{OTIMIZAÇÃO E VALIDAÇÃO DO MÉTODO}

Curvas analíticas foram construídas utilizando-se o método de padronização externa. Bons coeficientes de correlação $\left(r^{2}>0,99\right)$ foram obtidos nos intervalos de trabalho utilizados $(0,1$ a $\left.10,5 \mathrm{mg} \mathrm{L}^{-1}\right)$ para as substâncias estudadas, exceto para DEA $\left(r^{2}=0,9819\right)$ (Tabela 1).

\section{TABELA 1 - TEMPO DE RETENÇÃO $\left(t_{\mathrm{R}}\right)$, DADOS DA CURVA ANALÍTICA ( ${ }^{2}$, EQUAÇÃO, INTERVALO DE TRABALHO) E PRECISÃO INTRA-DIAS DAS SUBSTÂNCIAS ANALISADAS POR CGIDTE E CLAE/UV}

\begin{tabular}{lccccc}
\hline \multirow{2}{*}{ Substâncias } & \multicolumn{4}{c}{ Curva analítica } & Intervalo de \\
\cline { 2 - 5 } & $\mathrm{t}_{\mathrm{R}}(\mathrm{min})$ & $\mathrm{CV}(\%)$ & Equação & $\mathrm{R}^{2}$ & trabalho $\left(\mu \mathrm{mg} \mathrm{mL}^{-1}\right)$ \\
\hline DIA & 19,08 & 0,11 & $\mathrm{y}=119150 \mathrm{x}+772,41$ & 0,9943 & $0,1-4,1$ \\
\hline DEA & 19,61 & 0,14 & $\mathrm{y}=120706 \mathrm{x}+4541,5$ & 0,9819 & $0,1-4,1$ \\
\hline Trifluralina & 20,00 & 0,10 & $\mathrm{y}=40875 \mathrm{x}+20750$ & 0,9928 & $1,1-3,1$ \\
\hline Atrazina & 22,99 & 0,09 & $\mathrm{y}=162818 \mathrm{x}-9154,6$ & 0,9982 & $0,1-3,2$ \\
\hline Parationa metílica & 27,60 & 0,05 & $\mathrm{y}=616356 \mathrm{x}-37463$ & 0,9981 & $0,1-3,1$ \\
\hline 2,4-D & 5,27 & 0,59 & $\mathrm{y}=158885 \mathrm{x}-4680,4$ & 0,9988 & $0,1-10,0$ \\
\hline 2,4-DCF & 12,60 & 1,46 & $\mathrm{y}=142480 \mathrm{x}+8535,5$ & 0,9905 & $0,1-10,5$ \\
\hline
\end{tabular}

$\mathrm{CV}=$ coeficiente de variação para quatro replicatas.

Os parâmetros mais importantes para a análise de pesticidas em água por extração em fase sólida são o condicionamento do cartucho, a velocidade de eluição da amostra, o tempo de secagem do cartucho e a extração dos pesticidas retidos no adsorvente. Acetona, metanol e água são os solventes mais indicados para o condicionamento de cartuchos $C_{18}$ para análise do grupo de substâncias I (atrazina, desetilatrazina, deisopropilatrazina, trifluralina e parationa metílica) e II (2,4-D e 2,4-DCF), enquanto que para a extração, os mais utilizados são acetato de etila e diclorometano para o grupo I e metanol para o grupo II (PINTO e JARDIM, 2002; LAGANÀ et al., 2002; AMARANTE JUNIOR et al., 2002; VINK e VAN DER POLL, 1996; BUTZ; HEBERER e STAN, 1994; INCORVIA MATTINA, 1991). As propriedades físicas e químicas dos pesticidas também são importantes 
durante o desenvolvimento/otimização de métodos analíticos. Considerando a propriedade ácida do 2,4-D (pKa =2,64), o pH das amostras de água para análise do 2,4-D e do 2,4-DCF foi ajustado para 2. Em pH maior do que 2,6, as moléculas do 2,4-D encontram-se dissociadas, dificultando sua adsorção no adsorvente C18 (AMARANTE JUNIOR et al., 2002).

Em análise de resíduos de pesticidas, o método analítico é eficiente quando a recuperação do analito encontra-se na faixa entre $70-120 \%$, sendo considerado preciso quando o coeficiente de variação entre as amostras replicatas não ultrapassa 20\% (THIER e ZEUMER, 1987).

Os três sistemas de condicionamento avaliados resultaram em boa recuperação para atrazina, parationa metílica e DEA ( 90\%), entretanto baixos valores de recuperação foram observados para DIA e trifluralina $(<30 \%)$. Os solventes acetona, metanol e água, utilizados para condicionamento do cartucho e a velocidade de eluição da amostra igual a $8 \mathrm{~mL} \mathrm{~min}^{-1}$ resultaram em valores de recuperação satisfatórios para os pesticidas do grupo I, exceto para DIA. O tempo de secagem de 30 e 60 min evidenciaram os melhores resultados de recuperação, assim selecionou-se o tempo de $30 \mathrm{~min}$. A mistura acetato de etila:diclorometano (1:1, v/v) apresentou o melhor resultado de recuperação quando comparado ao acetato de etila e acetato de etila:n-hexano (7:3, v/v). A alta seletividade da mistura de solventes tornou-se necessária considerando as diferentes propriedades físicas e químicas dos pesticidas. Baixos valores de recuperação para as substâncias do grupo II foram observados (<47\%) nas velocidades de eluição da amostra de 3 e $5 \mathrm{~mL} \mathrm{~min}{ }^{-1}$. Os melhores resultados durante a otimização do método foram obtidos com a velocidade de pré-concentração da amostra e tempo de secagem do cartucho de $5 \mathrm{~mL} \mathrm{~min}^{-1}$ e $30 \mathrm{~min}$, respectivamente.

A exatidão (76-107\%) e a precisão ( $<12 \%)$ do método foram consideradas satisfatórias para a recuperação das substâncias nos dois níveis de fortificação estudados, exceto para DIA (38-51\%) (Tabela 2). Esse resultado pode ser justificado devido a sua elevada polaridade quando comparada às demais substâncias do grupo II. A seleção dos níveis de fortificação buscou atingir os valores máximos permitidos para essas substâncias em água para consumo humano, conforme estipulado pela legislação brasileira (BRASIL, 2006) em vigor (30 $\mathrm{g} \mathrm{L} \mathrm{L}^{-1}$ para 2,4-D, $2 \mu \mathrm{g} \mathrm{L}^{-1}$ para atrazina e $20 \mu \mathrm{g} \mathrm{L} \mathrm{L}^{-1}$ para trifluralina). Para os metabólitos e parationa metílica, não contemplados por essa legislação foram utilizados os valores da substância precursora e da atrazina, respectivamente.

\section{TABELA 2 - RECUPERAÇÃO, DESVIO PADRÃO E COEFICIENTE DE VARIAÇÃO DAS SUBSTÂNCIAS ANALISADAS}

\begin{tabular}{|c|c|c|c|c|c|c|}
\hline \multirow{2}{*}{ Substâncias } & \multirow{2}{*}{$\begin{array}{l}\text { Nível de fortificação } \\
\qquad\left(\mu g \mathrm{~L}^{-1}\right)\end{array}$} & \multicolumn{2}{|c|}{ Recuperação (\%) } & \multirow{2}{*}{$\begin{array}{l}\text { CV } \\
(\%)\end{array}$} & \multirow{2}{*}{$\begin{array}{l}\mathrm{LD} \\
\left(\mu g L^{-1}\right)\end{array}$} & \multirow{2}{*}{$\begin{array}{c}\mathrm{LQ} \\
\left(\mu \mathrm{gL}^{-1}\right)\end{array}$} \\
\hline & & Intervalo & Média* & & & \\
\hline \multirow{2}{*}{ DIA } & 1,0 & $44-51$ & 47 & 6 & \multirow[b]{2}{*}{-} & \multirow[b]{2}{*}{-} \\
\hline & 2,0 & $38-51$ & 45 & 11 & & \\
\hline \multirow{2}{*}{ DEA } & 1,0 & $79-100$ & 88 & 9 & \multirow{2}{*}{0,12} & \multirow{2}{*}{0,48} \\
\hline & 2,0 & $72-83$ & 76 & 5 & & \\
\hline \multirow{2}{*}{ Trifluralina } & 10,0 & $79-85$ & 82 & 3 & \multirow{2}{*}{0,10} & \multirow{2}{*}{0,30} \\
\hline & 20,0 & $71-80$ & 76 & 5 & & \\
\hline \multirow{2}{*}{ Atrazina } & 1,0 & $99-119$ & 107 & 7 & \multirow{2}{*}{0,084} & \multirow{2}{*}{0,25} \\
\hline & 2,0 & $102-106$ & 103 & 2 & & \\
\hline \multirow{2}{*}{ Parationa Metílica } & 1,0 & $91-101$ & 94 & 4 & \multirow{2}{*}{0,011} & \multirow{2}{*}{0,033} \\
\hline & 2,0 & 96-105 & 99 & 3 & & \\
\hline \multirow{2}{*}{$2,4-D$} & 10,0 & $103-111$ & 106 & 3 & \multirow{2}{*}{0,072} & \multirow{2}{*}{0,22} \\
\hline & 30,0 & 73-91 & 78 & 9 & & \\
\hline \multirow{2}{*}{ 2,4-DCF } & 10,0 & $79-88$ & 84 & 4 & \multirow{2}{*}{0,083} & \multirow{2}{*}{0,25} \\
\hline & 30,0 & $73-84$ & 77 & 5 & & \\
\hline
\end{tabular}

$C V$ = coeficiente de variação $(n=5) ; L D=$ limite de detecção do método; $L Q$ = limite de quantificação do método; - = não determinado. 
A ausência de picos interferentes nas amostras testemunhas (Figuras 2 e 3) confirma a seletividade do método proposto para determinação do grupo de substâncias I (atrazina, desetilatrazina, deisopropilatrazina, trifluralina e parationa metílica) e do grupo de substâncias II (2,4-D e 2,4-DCF). A escolha da fase sólida e dos solventes extratores resultou na eliminação de possíveis substâncias que pudessem interferir na identificação e quantificação das substâncias estudadas.
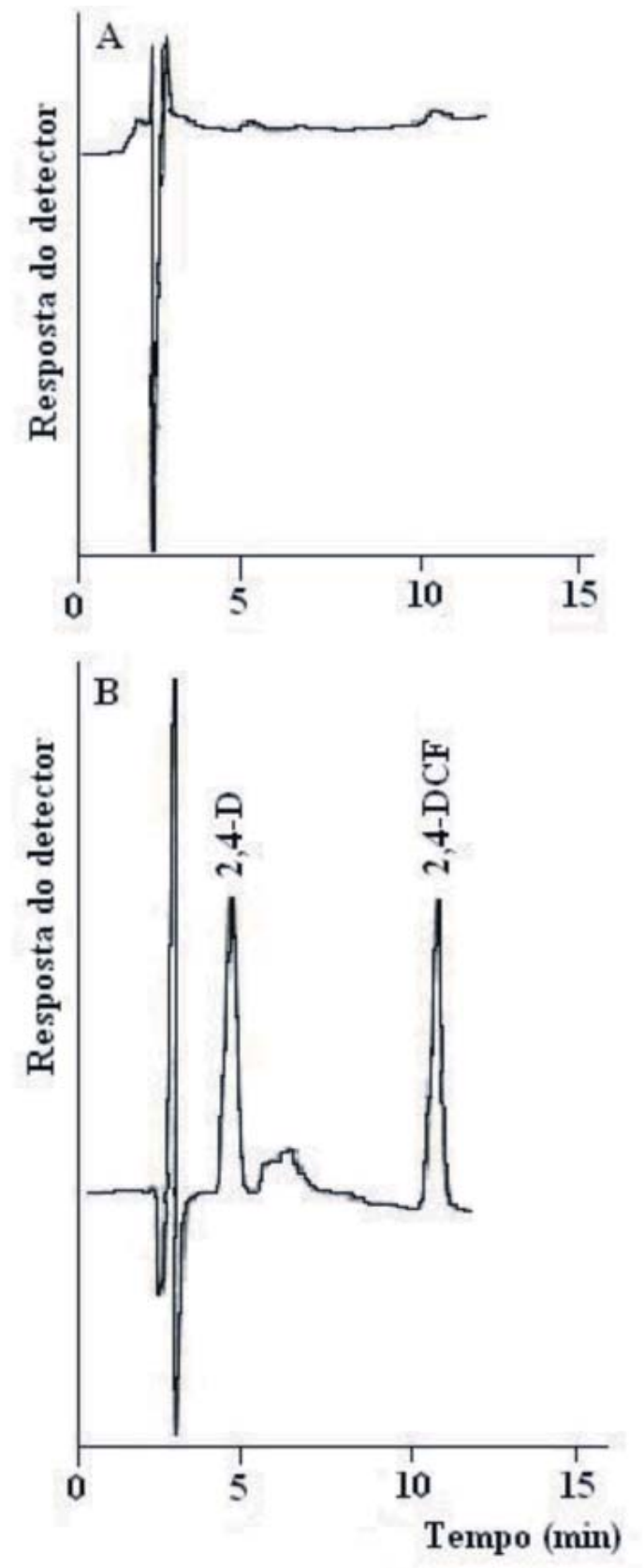

FIGURA 2 - CROMATOGRAMAS DA AMOSTRA TESTEMUNHA (A) E DA SOLUÇÃO PADRÃO DOS PESTICIDAS 2,4-D E 2,4-DCF (B) - RESPOSTA DO DETECTOR APRESENTADA NA MESMA ESCALA PARA AMBOS CROMATOGRAMAS (2,0 mg mL-1)

A avaliação da distribuição de resíduos de pesticidas em água requer normalmente a análise de grande número de amostras. Entretanto, nem sempre é possível analisar todas as amostras de água coletadas imediatamente para evitar alterações físicas, químicas e biológicas. 
Além do elevado número de amostras há a distância entre o ponto de coleta e o laboratório em que as análises cromatográficas são realizadas (SABIK e JEANNOT, 2000). Considerando que a técnica de EFS é muito utilizada para pré-concentração de substâncias em baixa concentração em água e o fato de que os cartuchos podem ser congelados e/ou transportados antes da etapa de extração, a avaliação da estabilidade dos analitos nos cartuchos é indicada (BARCELÓ e ALPENDURADA, 1996; AGUILAR BORRULL e MARCÉ, 1999).
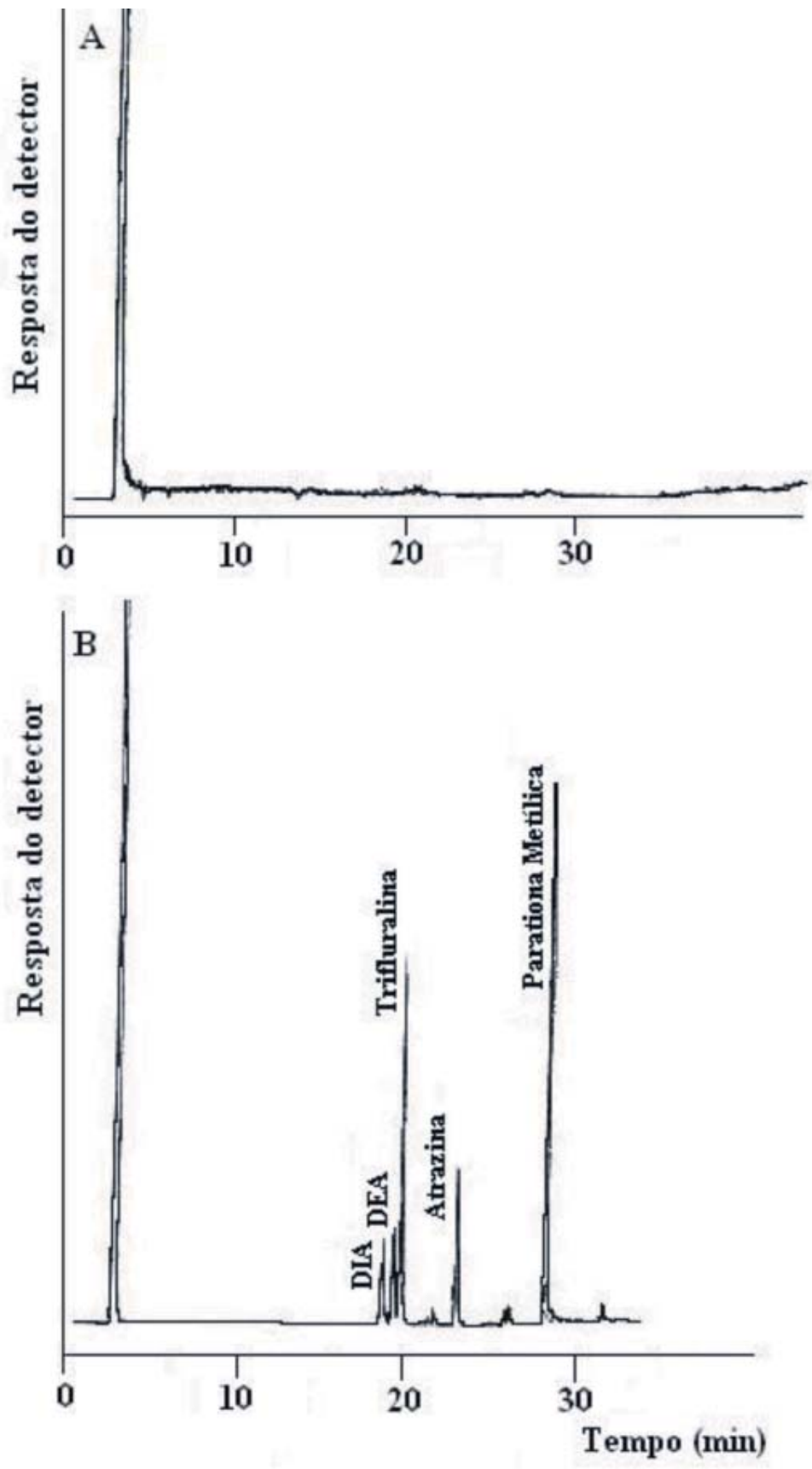

FIGURA 3 - CROMATOGRAMAS DA AMOSTRA TESTEMUNHA (A) E DA SOLUÇÃO PADRÃO DOS PESTICIDAS DIA (1,0 mg L-1), DEA (1,0 mg L-1), TRIFLURALINA (10,1 mg L-1),

ATRAZINA (1,1 mg L-1) E PARATIONA METÍLICA (1,0 mg L-1) (B) - RESPOSTA DO DETECTOR APRESENTADA NA MESMA ESCALA PARA 
Avaliou-se a estabilidade dos analitos estudados em cartuchos de EFS mantidos sob refrigeração $\left(-20^{\circ} \mathrm{C}\right)$ por 7,14 e 21 dias. Vinte e um dias foram considerados satisfatórios como intervalo entre a coleta das amostras, pré-concentração dos analitos em cartuchos de EFS (C18), a extração e sua análise cromatográfica.

Os resultados obtidos para os analitos quando submetidos ao congelamento, armazenamento e posterior eluição nos dois níveis de fortificação, situaram-se entre 78 e 105\% (CV < 6\%). Tal fato demonstra que as amostras após vinte e um dias da pré-concentração, armazenadas a $-20^{\circ} \mathrm{C}$, permanecem fidedignas, exceto o metabólito DIA que apresentou baixos valores de recuperação $(<51 \%)$. O fato dessa etapa ter sido realizada com água superficial também demonstra que não houve influência da matriz na eficiência dos métodos apresentados.

A literatura apresenta alguns trabalhos que abordaram a questão da conservação das amostras, após a pré-concentração de pesticidas em sistemas EFS (cartuchos ou discos). Entretanto, nenhum deles inclui o conjunto de condições investigadas no presente trabalho (moléculas, níveis de concentração e tipo de cartucho) (SABI e JEANNOT, 2000; AGUILAR BORRULL e MARCÉ, 1999).

O limite de detecção do método para os analitos estudados situou-se entre 0,011 e $0,12 \mu \mathrm{gL}^{-1}$ e o limite de quantificação entre 0,033 a $0,48 \mu \mathrm{g} \mathrm{L}^{-1}$, exceto para DIA, cujos valores não foram determinados porque o método não atendeu aos critérios de exatidão (Tabela 3 ). Essas concentrações estão de acordo com os valores máximos permitidos para os princípios ativos 2,4-D, atrazina e trifluralina em água potável, conforme a legislação vigente (BRASIL, 2006).

O grande diferencial da metodologia proposta, em relação àquelas descritas na literatura, refere-se à etapa do condicionamento dos cartuchos com combinação diferente de solventes que permitiu aumento significativo nos valores de recuperação, principalmente para o princípio ativo trifluralina (Tabela 2).

\subsection{APLICAÇÃO DO MÉTODO}

O herbicida 2,4-D foi a única substância encontrada acima do limite de detecção do método, tanto em água superficial como em subterrânea. As detecções, correspondentes a três (P3, P4 e P7) e cinco (P3, P4, P5, P6 e P8) pontos nos segundo e terceiro períodos de coleta, respectivamente, podem ser justificadas pelo seu elevado valor de solubilidade em água e baixo coeficiente de adsorção à matéria orgânica do solo em relação às demais substâncias estudadas. A não detecção dessa molécula no primeiro período de coleta justifica-se pela estiagem e ausência da aplicação efetiva dos pesticidas.

A comparação dos resultados obtidos com os apresentados na literatura para regiões de clima temperado e mesmo para regiões de clima tropical pode não ser a mais adequada, considerando as diferenças de solo, clima, relevo, propriedades do solo e dos aquíferos, intensidade de aplicação dos produtos e forma de manejo. No entanto, a apresentação de dados de outros autores que avaliaram a presença de pesticidas em amostras de água de diversos países (Tabela 3) confirma a relevância da geração de dados ambientais que possam subsidiar a elaboração do perfil da real contaminação dos recursos hídricos.

Deve-se ressaltar que alguns trabalhos encontrados na literatura não apresentam dados de validação de metodologia, o que é fundamental para dar confiabilidade aos resultados das investigações ambientais. 
TABELA 3 - DADOS DE VALIDAÇÃO E APLICAÇÃO DE MÉTODOS ANALÍTICOS PARA ANÁLISE DE ALGUNS DOS PESTICIDAS INVESTIGADOS NESTE TRABALHO EM ÁGUAS SUPERFICIAIS E SUBTERRÂNEAS

\begin{tabular}{|c|c|c|c|c|c|}
\hline Recuperação (\%) & $\operatorname{LD}\left(\mu \mathrm{g} \mathrm{L}^{-1}\right)$ & Água & Concentração $\left(\mu \mathrm{g} \mathrm{L}^{-1}\right)$ & País & Referência \\
\hline $\begin{array}{c}\text { Atrazina: } 87 \\
\text { Parationa metílica: } \\
84\end{array}$ & $\begin{array}{c}\text { Não } \\
\text { informado }\end{array}$ & Superficial & $\begin{array}{c}\text { Atrazina: } 0,12-5,16 \\
\text { Parationa metílica: } \\
\text { nd }-0,54\end{array}$ & China & GE et al., 2010 \\
\hline 2,4-D: 83 & 0,56 & Superficial & 2,4-D: $<0,56$ & China & FENG, ZHAO e LIN, 2009 \\
\hline $\begin{array}{c}\text { Atrazina }>80 \\
\text { DIA }>80 \\
\text { DEA }>80\end{array}$ & $\begin{array}{l}0,0006 \\
0,001 \\
0,003\end{array}$ & Superficial & $\begin{array}{c}\text { Atrazina: } 0,002- \\
0,048 \\
\text { DIA: } 0,002-0,012 \\
\text { DEA: } 0,019-0,479\end{array}$ & Canadá & GARCIA-AC et al., 2009 \\
\hline Não informado & $\begin{array}{c}\text { Não } \\
\text { informado }\end{array}$ & Superficial & $\begin{array}{c}\text { Atrazina: } 0,005- \\
5,505 \\
\text { DIA: } 0,001-0,013 \\
\text { DEA: } 0,002-0,027\end{array}$ & $\begin{array}{l}\text { Portugal } \\
\text { e } \\
\text { Espanha }\end{array}$ & PALMA et al., 2009 \\
\hline Atrazina $>60$ & 0,0036 & Superficial & $\begin{array}{c}\text { Atrazina: } 0,314- \\
0,901\end{array}$ & Espanha & KUSTER et al, 2008 \\
\hline $\begin{array}{l}\text { Parationa metílica: } \\
97\end{array}$ & 0,03 & Superficial & Parationa metílica: nd & China & WANG et al., 2007 \\
\hline $\begin{array}{c}\text { Atrazina: } 72 \\
\text { DEA: } 71 \\
\end{array}$ & $\begin{array}{c}0,00005 \\
0,0001 \\
\end{array}$ & Superficial & $\begin{array}{c}\text { Atrazina: nd - 0,0032 } \\
\text { DEA: nd }\end{array}$ & Itália & LOOS et al., 2007 \\
\hline $\begin{array}{l}\text { Atrazina: Não } \\
\text { informado }\end{array}$ & 0,1 & Superficial & Atrazina: nd - 0,48 & Brasil & BORTOLUZZI et al., 2007 \\
\hline Não informado & $\begin{array}{l}0,3 \\
0,3\end{array}$ & Superficial & $\begin{array}{c}\text { Trifluralina: nd } \\
\text { Atrazina: } 0,6-2,7\end{array}$ & Brasil & ARMAS et al., 2007. \\
\hline $\begin{array}{l}\text { Atrazina: } 102 \\
\text { DEA: } 94 \\
\text { Trifluralina: } 66\end{array}$ & $\begin{array}{c}\text { Faixa } \\
0,023- \\
0,088\end{array}$ & $\begin{array}{l}\text { Superficial } \\
\text { Subterrânea }\end{array}$ & $\begin{array}{c}\text { Atrazina: } 0,078 \text { - } \\
0,156 \\
\text { DEA: } 0,041-0,206 \\
\text { Trifluralina: } 0,068 \text { - } \\
0,102\end{array}$ & Brasil & DORES et al., 2006 \\
\hline Não informado & $\begin{array}{c}\text { Não } \\
\text { informado }\end{array}$ & Superficial & $\begin{array}{c}\text { Atrazina: } 0,0956 \mathrm{ng} \\
\mu \mathrm{L}^{-1}\end{array}$ & Brasil & $\begin{array}{c}\text { OCHI, BRITO e MEDEIROS, } \\
2010\end{array}$ \\
\hline Atrazina: 80 & 0,02 & Superficial & Atrazina: $0,03-0,09$ & Brasil & CERDEIRA et al., 2010 \\
\hline $\begin{array}{l}\text { Parationa metílica: } \\
62\end{array}$ & 0,020 & Subterrânea & Parationa metílica: nd & Índia & $\begin{array}{l}\text { SANKARARAMAKRISHNAN; } \\
\text { SHARMA e SANGHI, } 2005\end{array}$ \\
\hline $\begin{array}{c}\text { 2,4-D: } 99 \\
\text { Simazina: } 88 \\
\text { Carbendazim: } 98\end{array}$ & $\begin{array}{l}0,10 \\
0,10 \\
0,10\end{array}$ & Superficial & $\begin{array}{c}\text { 2,4-D: } 2,9-9,7 \\
\text { Simazina: nd - 3,0 } \\
\text { Carbendazim: } 0,4 \\
-4,5\end{array}$ & Chile & PALMA et al., 2004 \\
\hline $\begin{array}{c}\text { DEA: } 94 \\
\text { Atrazina: } 94\end{array}$ & $\begin{array}{l}0,0016 \\
0,0028\end{array}$ & $\begin{array}{l}\text { Superficial e } \\
\text { Subterrânea }\end{array}$ & $\begin{array}{l}\text { DEA: } 0,002-0,004 \\
\text { Atrazina:0,001-0,463 }\end{array}$ & Espanha & $\begin{array}{l}\text { RODRIGUES-MOZAZ; ALDA } \\
\text { e BARCELÓ, } 2004\end{array}$ \\
\hline $\begin{array}{c}\text { DEA: } 95 \\
\text { Trifluralina: } 105 \\
\text { Atrazina: } 110 \\
\text { Parationa Metílica: } \\
92\end{array}$ & $\begin{array}{l}0,010 \\
0,005 \\
0,005 \\
0,080\end{array}$ & Superficial & $\begin{array}{c}\text { DEA: 0,03 - 0,09 } \\
\text { Trifluralina:0,02 - 0,30 } \\
\text { Atrazina:0,02 - 0,23 } \\
\text { Parationa } \\
\text { Metílica:0,05 - 0,09 }\end{array}$ & Grécia & $\begin{array}{l}\text { LAMBROPOULOU et al., } \\
2002\end{array}$ \\
\hline $\begin{array}{l}\text { 2,4-D: } 98 \\
\text { 2,4-DCF: } 91\end{array}$ & $\begin{array}{l}0,001 \\
0,040\end{array}$ & Superficial & 2,4-D: nd - 0,5 & Itália & LAGANÀ et al., 2002 \\
\hline $\begin{array}{c}\text { Atrazina: } 95 \\
\text { 2,4-DCF: } 60 \\
\text { Parationa metílica: } \\
118 \\
\text { Trifluralina: } 56 \\
\text { DIA: } 50 \\
\text { DEA: } 86\end{array}$ & $\begin{array}{c}0,009 \\
0,02 \\
0,01 \\
0,005 \\
0,07 \\
0,01\end{array}$ & Superficial & $\begin{array}{c}\text { Atrazina:0,01 - 2,73 } \\
\text { DIA: 0,01-0,13 } \\
\text { DEA:0,01 - 0,5 }\end{array}$ & Portugal & AZEVEDO et al., 2000 \\
\hline $\begin{array}{c}\text { Trifluralina: } 96,7 \\
\text { Atrazina: } 80,6 \\
\text { Paratina metílica: } \\
109 \\
\end{array}$ & $\begin{array}{c}0,001 \\
0,002 \\
0,0005 \\
\end{array}$ & Superficial & $\begin{array}{c}\text { Atrazina:0,009-0,140 } \\
\text { Parationa metílica: } \\
\text { 0,021 -0,034 }\end{array}$ & Grécia & ALBANIS e HELA, 1995 \\
\hline
\end{tabular}

nd: não detectado. 


\section{CONCLUSÃO}

Os métodos apresentados neste trabalho requerem pequeno volume de solventes, são simples, precisos e exatos para as moléculas investigadas, exceto para DIA. Além disso, permitem detectar e quantificar as substâncias no nível estabelecido pela legislação nacional e pela Comunidade Européia. Embora os pesticidas selecionados não tenham sido detectados no período estudado, com exceção do 2,4-D, este trabalho alerta para a necessidade de avaliação sistemática da presença de pesticidas em água para consumo humano, particularmente, em regiões com intensa atividade agrícola, podendo fornecer subsídios para políticas públicas ambientais.

\section{ABSTRACT \\ VALIDATION AND APPLICATION OF METHOD FOR PESTICIDES ANALYSIS IN DRINKING WATER FROM DOURADOS (MS - BRAZIL) BY HPLC-UV AND GC-TSD}

Indiscriminate and inappropriate use of pesticides in agriculture has been pointed out for increasing health problems and environmental damage. Considering that water resources are the principal destiny of those compounds after application, the present study presents optimization and validation of two simple and efficient analytical methods for pesticides quantification in both surface and groundwater. Were selected the pesticides more commonly used at Dourados (MS - Brazil), region with intense agricultural activity. Pesticides were preconcentrated by solid-phase extraction using C18 $(500 \mathrm{mg})$ cartridges and then divided in two groups for elution and quantification: 2.4-D and 2.4-DCP were eluted with methanol and quantified by high performance liquid chromatography with ultra-violet detector (HPLC-UV) while atrazine, DIA, DEA, trifluralin and methyl parathion were eluted with ethylacetate $(1: 1, \mathrm{v} / \mathrm{v})$ and quantified by gas chromatography with thermionic specific detector (GC-TSD). The methods showed satisfactory accuracy $(76-107 \%)$ and precision $(<12 \%)$ for the substances analyzed at the fortified levels selected for the study, except for DIA $(<51 \%)$. Study of pesticide stability also presented good results: $\mathrm{C} 18$ cartridges could be stored for at least for 21 days at $-20^{\circ} \mathrm{C}$ with no signs of the compounds degradability. Both methods limits of quantification of the pesticides $\left(0.22-0.48 \mu \mathrm{g} \mathrm{L}^{-1}\right)$ are in accordance to the levels currently established by the Brazilian national legislation for pesticides in water. Although only the pesticide 2.4-D has been detected in two distinct collection points in the study period of time, this work warns for the requirement of systematical analysis of pesticides presence in water destined to human consume, principally in areas of intense agriculture activity. Such monitoring can provide subsidies for public environmental policies.

KEY-WORDS: VALIDATION; PESTICIDES; HPLC-UV; GC-TSD; DRINKING WATER.

\section{REFERÊNCIAS}

1 AGUILAR, C.; BORRULL, F.; MARCÉ, R. M. On-line and off-line solid-phase extraction with styrene-divinylbenzenemembrane extraction disks for determining pesticides in water by reversed-phase liquid chromatography-diode-array detection. Journal of Chromatography A, n. 754, p. 77-84, 1996.

2 ALBANIS, T. A.; HELA, D. G. Multi-residue pesticide analysis environmental water sample using solid-phase extraction disks and gas-chromatography with flame thermionic and mass-selective detection. Journal of Chromatography A, v. 707, n. 2, p. 283-292, 1995.

3 ALVES FILHO, J. P. Uso de agrotóxicos no Brasil: controle social e interesses corporativos. São Paulo: Annablume; Fapesp, 2002.

4 AMARANTE JUNIOR, O. P.; BRITO, N. M.; SANTOS, T. C. R.; RIBEIRO, M. L. Estudo da adsorção/dessorção de 2,4-D em solos usando técnica cromatográfica. Eclética Química, v. 27, p. 253-261, 2002.

5 ARMAS, E. D.; MONTEIRO, R. T. R.; ANTUNES, P. M.; SANTOS, M. A. P. F.; CAMARGO, P. B.; ABAKERLI, R. B. Diagnóstico espaço-temporal da ocorrência de herbicidas nas águas superficiais e sedimentos do rio Corumbataí e principais afluentes. Química Nova, v. 30, n. 5, p. 1119-1127, 2007.

6 AZEVEDO, D. A.; GERCHON, E.; REIS, E. O. Monitoring of pesticides and polycyclic aromatic hydrocarbons in water from Paraíba do Sul River, Brazil. Journal of the Brazilian Chermical Society, v. 15, n. 2, p. 292-299, 2004.

7 AZEVEDO, D. A.; LACORTE, S.; VINHAS, T.; VIANA, P.; BARCELÓ, D. Monitoring of pesticides and other organic pollutants in river water from Portugal by gas chromatography-mass spectrometry and liquid chromatography-atmospheric 
pressure chemical ionization mass spectrometry. Journal of Chromatography A, v. 879, p. 13-26, 2000.

8 BARCELO, D.; ALPENDURADA, M. F. A review of sample storage and preservation of polar pesticides in water samples. Chromatographia, v. 42, n. 11/12, p. 704-712, 1996.

9 BORTOLUZZI, E. C.; RHEINHEIMER, D. S.; GONÇALVES, C. S.; PELLEGRINI, J. B. R.; MARONEZE, A. M.; KURZ, M. H. S.; BACAR, N. M.; ZANELLA, R. Investigation of the occurrence of pesticide residues in rural wells and surface water following application to tobacco. Química Nova, v. 30, n.8, p. 1872-1876, 2007.

10 BRASIL. Ministério da Saúde. Portaria n 518, 25 de março de 2004. Estabelece os procedimentos e responsabilidades relativos ao controle e vigilância da qualidade da água para consumo humano e seu padrão de potabilidade, e dá outras providências. Disponível em: <http://www.agencia.pb.gov.br/arq/portarian_518mar2004.doc>. Acesso em: 10 fev. 2006.

11 BUTZ, S.; HEBERER, TH.; STAN, H. -J. Determination of phenoxyalkanoic acids and other acidic herbicides at the low ppt level in water applying solid-phase extraction with RP-C18 material. Journal of Chromatography A, v. 677, p. 63-74, 1994.

12 CERDEIRA, A. L.; PESSOA, M. C. P. Y.; SANTOS, N. A. G.; LANCHOTE, L. V. Lixiviação de atrazina em solo em área de recarga do aquífero Guarani. Disponível em: http://www.upf.br/rbherbicidas/download/RBH241.pdf, Acesso em: 10 maio 2010.

13 DALVIE, M. A.; SINANOVIC, E.; LONDON, L.; CAIRNCROSS, E.; SOLOMON, A.; ADAM, H. Cost analysis of ELISA, solidphase extraction, and solid-phase microextraction for the monitoring of pesticides in water. Environmental Research, $v$. 98, p. 143-150, 2005.

14 DORES,E. F. G. C.; NAVICKIENE, S.; CUNHA, M. L. F.; CARBO, L.; RIBEIRO, M. L.; DE-LAMONICA-FREIRE, E. M. L. Multiresidue determination of herbicides in environmental waters from Primavera do Leste region (Middle West of Brazil) by SPE-GC-NPD. Journal of the Brazilian Chemical Society, v. 17, n. 2, p. 866-873, 2006.

15 DORES, E. F. G. C.; DE-LAMONICA-FREIRE, E. M. Contaminação do ambiente aquático por pesticidas, estudo de caso: águas usadas para consumo humano em Primavera do Leste, Mato Grosso - análise preliminar. Química Nova, v. 24, n.1, p. 27-36, 2001.

16 FENG, Q.; ZHAO, L.; LIN, J-M. Molecularly imprinted polymer as micro-solid phase extraction combined with high performance liquid chromatography to determine phenolic compounds in environmental water samples. Analytica Chimica Acta, v. 650, p. 70-76, 2009.

17 FILIZOLA, H. F.; FERRACINI, V. L.; SANS, L. M. A.; GOMES, M. A. F.; FERREIRA, C. J. A. Monitoramento e avaliação do risco de contaminação por pesticidas em água superficial e subterrânea na região de Guairá. Pesquisa Agropecuaria Brasileira, v. 37, n. 5, p. 659-667, 2002.

18 FRITZ, J.S. Solid-phase extraction. In: LAURENT, G.; SHAPIRO, S. (Eds.) Encyclopedia of analytical science. $2^{\text {nd }}$ ed. Amsterdam: Elsevier, 2005.

19 GARCIA-AC, A.; SEGURA, P. A.; VIGLINO, L.; FÜRTÖS, A.; GAGNON, C.; PRÉVOST, M.; SAUVÉ, S. On-line solid-phase extraction of large-volume injections coupled to liquid chromatography-tandem mass spectrometry for the quantization and confirmation of 14 selected trace organic contaminants in drinking and surface water. Journal of Chromatography A, v. 1216, p. 8518-8527, 2009.

20 GE, J. ; CONG, J.; SUN, Y.; LI, G.; ZHOU, Z.; QIAN, C.; LIU, F. Determination of endocrine disrupting chemicals in surface water and industrial wastewater from Beijing, China. Bulletin of Environmental Contamination and Toxicology, v. 84, p. 401-405, 2010.

21 GONÇALVES, C.; ALPENDURADA, M. F. Comparison of three different poly(dimethylsiloxane) - divinylbenzene fibres for the analysis of pesticide multiresidues in water samples: structure and efficiency. Journal of Chromatography A, v. 963, p. 19-26, 2002

22 INCORVIA MATTINA, M. J. Determination of chlorophenoxy acids using highperformance liquid chromatography-particle beam mass spectrometry. Journal of Chromatography A, v. 542, p. 385-395, 1991.

23 KUSTER, M.; DE ALDA, M. J. L.; BARATA, C.; RALDÚA, D.; BARCELÓ, D. Analysis of 17 polar to semi-polar pesticides in the Ebro river delta during the main growing season of rice by automated on-line solid-phase extraction-liquid chromatography-tandem mass spectrometry. Talanta, v. 75, p. 390-401, 2008.

24 LAGANÀ, A.; BACALONI, A.; DE LEVA, I.; FABERI, A.; FAGO, G.; MARINO, A. Occurrence and determination of herbicides and their major transformation products in environmental waters. Analytica Chimica Acta, v. 462, p. 187-198, 2002.

25 LAMBROPOULOU, D. A.; SAKKAS, V. A.; HELA, D. G.; ALBANIS, T. A. Application of solid-phase microextraction in the monitoring of priority pesticides in the Kalamas River (N.W. Greece). Journal of Chromatography A, v. 963, p. 107-116, 2002.

26 LOOS, R.; WOLLGAST, J.; HUBER, T.; HANKE, G. Polar herbicides, pharmaceutical products, perfluorooctanesulfonate 
(PFOS), perfluorooctanoate (PFOA), and nonylphenol and its carboxylates and ethoxylates in surface and tap waters around Lake Maggiore in Northern Italy. Analytical and Bioanalytical Chemistry, v. 387, p. 1469-1478, 2007.

27 LOPES, M. N. T. Efeito de sistemas de manejo de solo e de doses de nitrogênio em interação pastagem/soja. 2001. 60 f. Dissertação (Mestrado em Agronomia) - Faculdade de Ciências Agronômicas, Universidade Estadual Paulista, Botucatu, 2001.

28 MA, W. T.; FU, K. K.; CAI, Z.; JIANG, G. B. Gas chromatography/mass spectrometry applied for the analysis of triazine herbicides in environmental waters. Chemosphere, v. 52, p. 1627-1632, 2003.

29 MENOR-HIGUERUELO, S.; PÉREZ-ARRIBAS, L. V.; LEÓN-GONZÁLES, M. E.; POLO-DÍEZ, L. M. Solid-phase extraction of chlorophenoxy acid herbicides by means of polymeric resins functionalized with quaternary ammonium groups. Journal of Liquid Chromatography \& Related Technologies, v. 25, n. 3, p. 445-461, 2002

30 OCHI, B. C.; BRITO, V. C.; MEDEIROS, M. A. C. Avaliação da contaminação de águas superficiais por herbicidas e nutrientes e em áreas com o cultivo de cana-de-açúcar no município de Iracemápolis, SP, Brasil. Disponível em: http://www.cori.unicamp.br/jornadas/completos/UNICAMP/AVALIAcaO\%20DA\%20CONTAMINAcaO\%20DE.doc. Acesso em: 10 maio 2010.

31 PALMA, P.; KUSTER, M.; ALVARENGA, P.; PALMA, V. L.; FERNANDES, R. M.; SOARES, A. M. V. M.; DE ALDA, M. J. L.; BARCELÓ, D.; BARBOSA, I. R. Risk assessment of representative and priority pesticides, in surface water of the Alqueva reservoir (South of Portugal) using on-line solid phase extraction-liquid chromatography tandem mass spectrometry. Environment International, v. 35, p. 545-551, 2009.

32 PALMA, G.; SANCHEZ, A.; OLAVE, Y.; ENCINA, F.; PALMA, R.; BARRA, R. Pesticide levels in surface waters in an agricultural-forestry basin in Southern Chile. Chemosphere, v. 57, p. 763-77, 2004.

33 PAPADOPOULOU-MOURKIDOU, E.; KARPOUZAS, D. G.; PATSIAS, J.; KOTOPOULOU, A.; MILOTHRIDOU, A.; KINTZIKOGLOU, K.; VLACHOU, P. The potential of pesticides to contaminate the groundwater resources of the Axios river basin in Macedonia, Northern Greece. Part I.Monitoring study in the north part of the basin. Science of the Total Environment, v. 321, p. 127-146, 2004a.

34 PAPADOPOULOU-MOURKIDOU, E.; KARPOUZAS, D. G.; PATSIAS, J.; KOTOPOULOU, A.; MILOTHRIDOU, A.; KINTZIKOGLOU, K.; VLACHOU, P. The potential of pesticides to contaminate the groundwater resources of the Axios river basin.Part II.Monitoring study in the south part of the basin. Science of the Total Environment, v. 321, p. 147-164, 2004b.

35 PINTO, G. M. F.; JARDIM, I. C. S. F. Use of solid phase extraction and HPLC for determination of herbicide multiresidue recoveries in water. Journal of Liquid Chromatography \& Related Technologies, v. 25, n. 7, p. 1093-1101, 2002.

36 RIBEIRO, M. L.; LOURENCETTI, C.; PEREIRA, S. Y.; DE MARCHI, M. R. R. Contaminação de águas subterrâneas por pesticidas: avaliação preliminar. Química Nova, v. 30, n. 3, p. 668-694, 2007.

37 RODRIGUES-MOZAZ, S.; ALDA, M. J. L.; BARCELÓ, D. Monitoring of estrogens, pesticides and bisphenol A in natural waters and drinking water treatment plants by solidphase extraction-liquid chromatography-mass spectrometry. Journal of Chromatography A, v. 1045, p. 85-92, 2004.

38 SABIK, H.; JEANNOT, R. Stability of organophosphorus insecticides on graphitized carbon black extraction cartridges used for large volumes of surface water. Journal of Chromatography A, v. 879, p. 73-82, 2000.

39 SANKARARAMAKRISHNAN, N.; SHARMA, A. K.; SANGHI, R. Organochlorine and organophosphorous pesticide residues in ground water and surface waters of Kanpur, Uttar Pradesh, India. Environment International, v. 31, p. 113$120,2005$.

40 SPADOTTO , C. A.; GOMES, M. A. F.; LUCHINI, L. C.; ANDÉA, M. M. Monitoramento de risco ambiental de agrotóxicos: princípios e recomendações. Jaguariúna: Embrapa Meio Ambiente, 2004. 29 p. (Documento, 42).

41 SPRINGWAY. Aágua no mundo. Disponível em: http://www.springway.com.br/aagu/aamu.html. Acesso em: 23 mar. 2005.

42 THIER, H. P.; ZEUMER, H. Manual of pesticide analysis. New York: Verlag Chemie, 1987. 433 p.

43 TUNDISI, J. G. Planejamento e gestão dos recursos hídricos: novas abordagens e tecnologias. In: século XXI: enfrentando a escassez. São Carlos: RiMa, 2003a. cap. 7. p. 105-137.

Água no

44 TUNDISI, J. G. Usos múltiplos das águas superficiais e subterrâneas. In: Água no século XXI: enfrentando a escassez. São Carlos: RiMa, 2003b. cap. 3. p. 27-34.

45 VINK, M.; VAN DER POLL, J. M. Gas chromatographic determination of acid herbicides in surface water samples with electron-capture detection and mass spectrometric. Journal of Chromatography A, v. 733, p. 361-366, 1996.

46 WANG, S.; ZHAO, P.; MIN, G.; FANG, G. J. Multi-residue determination of pesticides in water using multi-walled carbon nanotubes solid-phase extraction and gas chromatography-mass spectrometry. Journal of Chromatography A, v. 1165, p. 166-171, 2007. 